\title{
Game Design for Older Adults: Effects of Age-Related Changes on Structural Elements of Digital Games
}

\author{
Kathrin Maria Gerling ${ }^{1}$, Frank Paul Schulte ${ }^{2}$, Jan Smeddinck ${ }^{3}$, and Maic Masuch ${ }^{2}$ \\ ${ }^{1}$ University of Saskatchewan, 110 Science Place, \\ Saskatoon SK S7N 5C9, Canada \\ kathrin.gerling@acm.org \\ ${ }^{2}$ University of Duisburg-Essen, Forsthausweg 2, \\ 47057 Duisburg, Germany \\ \{frank.schulte, maic.masuch\}@uni-due.de \\ ${ }^{3}$ University of Bremen, Bibliothekstraße 1, \\ 28359 Bremen, Germany \\ smeddinck@tzi.de
}

\begin{abstract}
Recent studies report various positive effects on elderly persons playing digital games. Yet, games are rarely designed with an elderly user group in mind. In this paper, this issue is addressed by providing an overview of common age-related changes followed by a summary of game design considerations for senior audiences. The impact of age on game design is discussed based on an analysis of the most important structural elements of games. The analysis shows that age-related changes in users' cognitive and physical abilities affect the use of games on multiple levels, making the complexity of games and interrelations between different game mechanics a crucial factor when designing for older adults.
\end{abstract}

Keywords: Game design, older adults, accessibility, design recommendations.

\section{Introduction}

Engaging with digital games has a positive impact on the emotional and physical well-being of elderly in nursing homes as recent research suggests [10]. However, many commercially available gaming products are not suitable for elderly persons [7]. Apart from the development of therapeutic applications and serious games, a structured approach to digital game design for an elderly audience based on agerelated changes and impairments has rarely been the subject of academic work [11]. Understanding both chances and challenges of an elderly target audience is vital, if senior citizens are to be addressed by and engaged in video games [9]. Core aspects that might foster or hinder the engagement of seniors in the use of video games as leisure activity must be identified. This paper provides an overview of age-related changes and diseases and discusses existing approaches towards game design for senior citizens, which are extended based on a structural analysis of digital games. 


\section{Game Design for Older Adults}

This section summarizes age-related needs and changes that are caused by regular aging processes and provides an overview of game design recommendations to foster the creation of accessible games for older adults.

\subsection{Common Age-Related Changes Among Senior Citizens}

From a biopsychological perspective, aging affects the quality of life on various levels. Besides sensory decrements in vision and hearing [7], older adults often experience:

- Cognitive impairments, which affect problem solving skills, information processing $[2,6]$ and result in a reduced attention span when working on complex tasks $[2,3]$.

- A decline of motor skills, which includes a decrease in fine motor skills, changes in posture and balance [2], and negatively affect motor learning [2, 3].

- Chronic illnesses, which range from arthritis to severe heart conditions, and which have an impact on the physical abilities and mobility of senior citizens [3].

Older adults living in full-care nursing homes are most likely to be severely influenced by age-related impairments [3]. Additional diseases may lead to more physical impairments in late life. Generally speaking, age-related changes limit the patient's possibilities of participating in regular leisure activities and lead to special requirements for the design of interactive entertainment systems.

\subsection{Game Design Considerations}

In the early 80s, Weisman introduced video games to institutionalized older adults [15]: Based on observations, he claims that games with an adaptable level of difficulty are advantageous because they support individual preferences and sensomotor abilities best. The use of large and well-defined visual symbols and clear auditory feedback is recommended to address visual and auditory impairments. Almost 25 years later, Ijsselsteijn et al. [9] identify the decline of visual and auditory abilities as well as a loss of sensomotor skills as most important age-related impairments, which influence the use and reception of digital games by senior citizens. They suggest visually adjustable games (e.g. regarding the font- and window-size, colors and contrast) that deliver multimodal feedback relying on more than one communication channel and feature user interfaces that are of low complexity. Flores et al. [4] include the design of appropriate cognitive challenges, which have the potential of keeping the interest of older adults. Based on findings from the ElderGames project, usability criteria for advanced technologies for the elderly were compiled [6]. They include a reduction of steps necessary to complete 
tasks, a reduction of the cognitive load, the availability of immediate feedback and the adaptation of digital systems to the users' goals. Additionally, the importance of system consistency and fault tolerance is highlighted.

Furthermore, when designing entertainment systems for senior citizens, it seems important to consider the impact of game content, genres and benefits from playing digital games. Ijsselsteijn et al. [9] identified games for relaxation and entertainment, games which support social activities, games to sharpen one's mind, and exertion games as suitable game types. Flores et al. [4] highlight the creation of meaningful play through learning objectives and social play. Results from the ElderGames project suggest that elderly players have a preference for simplistic puzzle and quiz games, which may have a positive impact on daily life [9, 13]. De Schutter and Vanden Abeele [13] identified connecting players, cultivating skills and contributing to society as important aspects of digital games for older adults. In terms of game features, the social aspect of games is frequently addressed by the recommendation of a multiplayer mode. A large amount of senior citizens engages in games as active non-players who watch others play, cheer and comment on in-game situations [14].

The guidelines and design recommendations presented within this section cover the issue of aging and game design on a formal level and primarily address usability and accessibility issues. The majority of guidelines focuses on the impact of age-related changes and impairments on interface design and includes detailed recommendations for designers. However, those design guidelines that address game features and genres only discuss game design issues on a meta-level (e.g. in terms of genre recommendations) and do not give detailed information on the impact of age on structural elements of games.

\section{Structural Models of Games}

This section provides an overview of two theoretical approaches to a formal description of digital games by Adams [1] and Fullerton [5]. For an overview of the development of the definition of games cf. Salen and Zimmerman [12].

Fullerton's [5] formal elements of games include players, objectives, procedures, rules, resources, conflict, boundaries and an outcome. Adams' [1] structured approach towards the key components of video games primarily focuses on the interaction between core game mechanics and the user interface. Core mechanics include in-game resources, further attributes as well as general mechanics [1]. The user interface acts as a broker between core mechanics and the player. Because of the strong focus on interface design and its relation with game mechanics, Adams' model offers the opportunity of combining interface-related design guidelines with complex criteria derived from age-related needs and requirements which are expected to map on the complexity of digital games. In contrast, Fullerton provides a broad approach towards the structure of games that covers relevant areas of game design. 


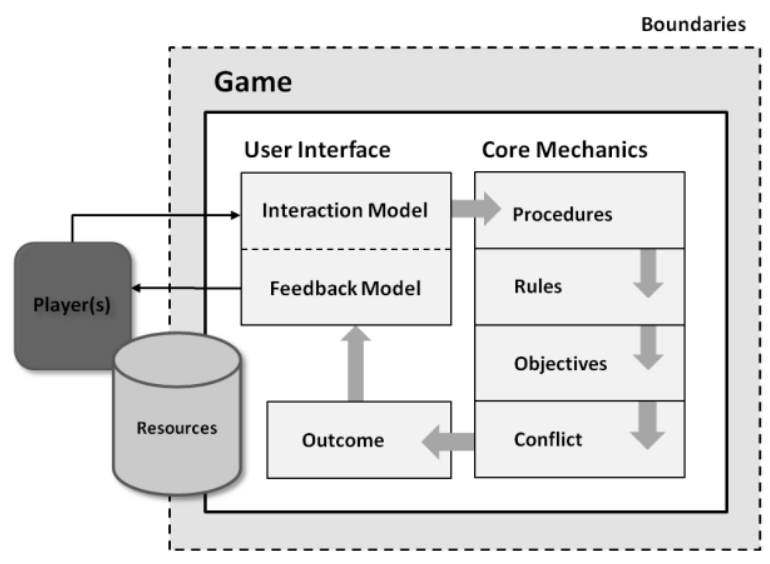

Fig. 1. The extended model of digital games (based on Adams and Fullerton)

\subsection{Creation of an Extended Model}

Both Adams and Fullerton refer to games as complex systems that feature different sets of mechanics to create a unique user experience. These mechanics are affected by user input. According to Adams, the interaction model turns user input into actions, which may be interpreted by the core mechanics of the game [1]. Fullerton refers to actions as a part of procedures that are deeply rooted within the rules of the game, but does not define the precise relationship between user interface and game mechanics. Additionally, her concept of procedures includes both player actions and system procedures, which incorporate complex methods of play [5]. While Fullerton suggests that the outcome of the game affects the player and determines whether the conflict was successfully resolved, Adams states that new challenges may be presented to the player via the user interface. Because Fullerton does not give detailed information regarding the relationship between game mechanics and the user interface, Adams' model offers the possibility of extending her definition. Other than that, Adams' definition of core mechanics is not as precise as Fullerton's description of the structure of games. In the extended model, Adams' camera model is referred to as feedback model, including visual, haptic and auditory output. Figure 1 shows a combination of the approaches.

\subsection{Effects of Age-Related Changes on Structural Models of Games}

Based on the joint model, the relevance of age-related changes and age-associated diseases in the context of game design can be analyzed with a focus on the impact of age on the structural elements of games.

Players and Resources. When designing for today's senior audience, game designers have to create games for a target group with - at least nowadays - very limited gaming experience. Since research has shown that elderly persons enjoy both 
engaging in active play as well as following games as spectators and commenting on other players, designers should address the needs of those audience participants, e.g. by offering interesting visual sceneries, or fostering competition similar to sports events. Depending on the context of play, different competition types seem appropriate for elderly persons. "Local team versus game" competitions offer the opportunity of engaging groups of senior citizens in play, online multiplayer functionality offers the possibility of experiencing competition or cooperation to senior citizens who live an independent life. Resources are another important aspect that should be considered. The attention span of the player may be a relevant resource: When games require elaborate decision making or quick reactions, changes in short-term memory might affect the player's ability to recall in-game information at a later point. Sensorimotor skills are an important resource if certain game mechanics include complex interaction paradigms or repetitive user input and timecritical player feedback. Generally, the availability of in-game resources should not be as restrictive as for a younger audience and ideally be individually adjustable.

User Interface. The use of input devices may also be limited due to age-related changes in motor skills. While active senior citizens may still be able to use regular game pads, institutionalized older adults may experience difficulties in holding the controller in their hands while pressing small buttons. Furthermore, the complexity of input sequences should be significantly reduced for an elderly audience to account for decrements of cognitive abilities, especially attention processes and short-term memory. In this context, possible difficulties with parallel player input (e.g. moving a haptic device and pressing a button at a certain point of time or performing complex point-and-click operations) should be considered. Additionally, the graphical user interface has to be adjusted to meet the needs of elderly persons (e.g. with regard to visual game element size, font size, high contrast, etc.) when designing the feedback model which presents the game to the player, for instance via visual, auditory or haptic output.

Core Mechanics. Core mechanics describe internal processes and requirements of digital games. They consist of procedures, rules, objectives and conflict and define both challenges of and players' interactions with the game. Certain aspects of core mechanics (e.g. limitations caused by rules) have to be learned in order to master a game. Decrements in memory and elderly persons' attention span affect information processing from short-term to procedural memory. Thus, the ability of learning how to play a game is limited, and learning processes take more time if the player is affected.

In-game actions available to the player - procedures - should be easy to understand and ideally incorporate metaphors of daily life to facilitate learning processes. Additionally, designers should avoid parallel input and quick sequential reactions to lower the player's cognitive load and account for changes in sensorimotor abilities. Rules should be easy to understand to facilitate the player's entry into game play. In this context, elderly players may need additional and more detailed information to understand restrictions and requirements of digital games due to their lack of gaming experience. Objectives have to be more clearly defined and 
communicated to the elderly player. Since objectives have an impact on the overall direction on the game, it is not only important to account for age-related changes, but also to consider suitable game genres and features.

Outcome. Because age-related impairments are likely to reduce the player's ability to master games in comparison to a younger audience, it is important to consider the effects of impairments when defining the outcome of the game. Adjustable limits for the internal assessment of player success offer the possibility of providing the user with feedback which is based on his or her individual skills.

\section{$4 \quad$ Application of the Model}

Based on the evaluation results [8] and observations during the design process of SilverPromenade, a game specifically addressing older adults, we discuss the applicability of the extended model of digital games in game design for senior citizens and potential extensions and future use-cases.

When creating the game design concept for SilverPromenade, we applied the extended model of digital games at an early stage of the development process. First, we created a general vision outline of the game concept. Second, we redefined single aspects using the list of structural elements and the associated recommendations regarding the impact of age. The model helped shape our game design document and provided a foundation for the more detailed analysis of certain game design decisions, the discussion of design alternatives and the integration of suitable game mechanics, e.g. the inclusion of the three different player roles to accommodate different abilities. Finally, when preparing the evaluation of the SilverPromenade, the model could be used to draw attention to important aspects of the game and provided a foundation for observations during the study. On a general level, the extended model of digital games with a focus on age-related changes provides a structure for designers to consider important issues associated with senior audiences during the design process. Its broad approach can help them to encompass basic needs of older adults and foster the creation of accessible games; the model embraces a wide range of game design elements supporting the systematic examination of the impact of ageing on the interaction with video games. In contrast to previous work on game design recommendations for elderly persons, the model accounts for the connection of interface and game design by linking the effects of core mechanics as well as the interaction and feedback model. Recognizing interrelations between user interfaces and game mechanics is also important when considering the growing popularity of motion-based games, in which interaction paradigms are frequently matched with game mechanics and which hold the promise of fostering physical activity.

Limitations of the Model. Even when accounting for common age-related changes, older adults represent a heterogeneous target audience, thus general player abilities are difficult to capture. The evaluation of SilverPromenade showed that although our model covers the most important issues of game design for elderly, it lacks individual details that are difficult to integrate. To solve this issue, we suggest a user-centered 
design process which allows for user-testing at an early stage for further adjustments. Additionally, the current version of the model accounts for technical and structural aspects of game design only. In order to fully grasp the interaction between players and the game, the aspect of user experience needs to be examined and integrated into the existing approach. This is especially interesting when designing for a senior audience as only little research focused on the game experience of elderly players. Another opportunity which has not been explored and that could be examined based on structural considerations is the design of narrative and dramatic elements in video games. Generally speaking, the model presented in this paper provides a broad overview of common age-related issues as a starting point for further considerations. Individual adjustments have to be made based on an analysis of the particular target audience and application context to ensure the accessibility.

\section{Conclusion and Future Work}

In this paper, the impact of prevalent age-related impairments among senior citizens on structural elements of video games was discussed based on a joint model of digital games featuring the work of Adams [1] and Fullerton [5], which was then evaluated through the design of SilverPromenade [8]. The analysis showed that cognitive and sensory decrements as well as age-associated diseases affect various aspects regarding the use of digital games and have to be considered when designing for older adult players. While sensory decrements mostly affect the design of the user interface and appropriate design considerations have been presented by many authors, cognitive impairments and the lack of gaming experience among today's elderly population are an important factor when designing game mechanics. In this context, the analysis has shown that the complexity of digital games and interrelations between different mechanics are a crucial factor when designing for older adults: On the one hand, elderly players have to be provided with sufficient information to be able to interact with the game adequately. On the other hand, core mechanics of the game have to be simplistic and easy to learn in order to reduce the cognitive load of an elderly audience. A structured analysis of digital games and their interrelation with agerelated changes helps fostering the integration of concerns regarding the target audience early in the development process. It may also help to guide designers even if they have no prior experience in working with elderly. Future work includes the further verification of the feasibility of design recommendations based on the structural approach towards digital games presented in this paper. We aim to examine the usage of our model beyond academic game development to gain further insights into the applicability of our work in an industry context.

\section{References}

[1] Adams, E.: Fundamentals of Game Design. New Riders Publishing, Berkeley (2010)

[2] Birren, J.E., Schaie, K.W.: Handbook of the Psychology of Aging. Academic Press, San Diego (2001) 
[3] Czaja, S.J., Lee, C.C.: Information Technology and Older Adults. In: Sears, A., Jacko, J.A. (eds.) The Human-Computer Interaction Handbook. Lawrence Erlbaum Associates, New York and London (2006)

[4] Flores, E., Tobon, G., Cavallaro, E., Cavallaro, F.I., Perry, J.C., Keller, T.: Improving patient motivation in game development for motor deficit rehabilitation. In: Proceedings of ACE 2008, Yokohama, Japan (2008)

[5] Fullerton, T.: Game Design Workshop, Second Edition: A Playcentric Approach to Creating Innovative Games. Morgan Kaufmann Publishers, Burlington (2008)

[6] Gamberini, L., Alcaniz, M., Barresi, G., Fabregat, M., Ibanez, F., Prontu, L.: Cognition, technology and games for the elderly: An introduction to ELDERGAMES Project. PsychNology Journal 4(3), 285-308 (2006)

[7] Gerling, K.M., Schild, J., Masuch, M.: Exergame Design for Elderly Users: The Case Study of SilverBalance. In: Proceedings of ACE 2010, Taipei, Taiwan (2010)

[8] Gerling, K.M., Schulte, F.P., Masuch, M.: Designing and Evaluating Digital Games for Older adults. In: Proceedings of ACE 2011, Lisbon, Portugal (2011)

[9] Ijsselsteijn, W., Nap, H.H., de Kort, Y.: Digital Game Design for Elderly Users. In: Proceedings of FuturePlay 2007, Toronto, Canada (2007)

[10] Jung, Y., Li, K.J., Janissa, N.S., Gladys, W.L.C., Lee, K.M.: Games for a Better Life: Effects of Playing Wii Games on the Well-Being of Seniors in a Long-Term Care Facility. In: Proceedings of IE 2009, Sydney, Australia (2009)

[11] Nap, H.H., de Kort, Y.A.W., IJsselsteijn, W.A.: Senior gamers: preferences, motivations and needs. Gerontechnology 8(4), 247-262 (2009)

[12] Salen, K., Zimmerman, E.: Rules of Play: Game Design Fundamentals. MIT Press, Cambridge (2004)

[13] De Schutter, B., Vanden Abeele, V.: Meaningful Play in Elderly Life. In: Proceedings of the 58th Annual Conference of the ICA, Montreal, Canada (2008)

[14] Shim, N., Baecker, R., Birnhotz, J., Moffatt, K.: TableTalk Poker: An Online Social Gaming Environment for Seniors. In: Proc. of FuturePlay 2010 (2010)

[15] Weisman, S.: Computer Games for the Frail Elderly. Gerontologist 23(4), 361-363 (1983) 